\title{
Cbl negatively regulates JNK activation and cell death
}

\author{
Andrew A Sproul ${ }^{1,2}$, Zhiheng $\mathrm{Xu}^{5}$, Michael Wilhelm ${ }^{3}$, Stephen Gire ${ }^{4}$, Lloyd A Greene ${ }^{2}$ \\ ${ }^{1}$ Department of Biological Sciences, Columbia University, New York, New York, USA; ${ }^{2}$ Department of Pathology and Cell Biology, \\ Columbia University, New York, New York, USA; ${ }^{3}$ Department of Pediatrics, University of Wisconsin Madison-School of Medicine \\ and Public Health, Madison, Wisconsin, USA, ${ }^{4}$ Department of Pediatrics, Columbia University, New York, New York, USA; ${ }^{5}$ Key \\ Laboratory of Molecular and Developmental Biology, Institute of Genetics and Developmental Biology, Chinese Academy of Sci- \\ ences, Beijing, China
}

Here, we explore the role of $\mathrm{Cbl}$ proteins in regulation of neuronal apoptosis. In two paradigms of neuron apoptosis - nerve growth factor (NGF) deprivation and DNA damage - cellular levels of c-Cbl and Cbl-b fell well before the onset of cell death. NGF deprivation also induced rapid loss of tyrosine phosphorylation (and most likely, activation) of c-Cbl. Targeting c-Cbl and Cbl-b with siRNAs to mimic their loss/inactivation sensitized neuronal cells to death promoted by NGF deprivation or DNA damage. One potential mechanism by which Cbl proteins might affect neuronal death is by regulation of apoptotic c-Jun N-terminal kinase (JNK) signaling. We demonstrate that Cbl proteins interact with the JNK pathway components mixed lineage kinase (MLK) 3 and POSH and that knockdown of Cbl proteins is sufficient to increase JNK pathway activity. Furthermore, expression of c-Cbl blocks the ability of MLKs to signal to downstream components of the kinase cascade leading to JNK activation and protects neuronal cells from death induced by MLKs, but not from downstream JNK activators. On the basis of these findings, we propose that Cbls suppress cell death in healthy neurons at least in part by inhibiting the ability of MLKs to activate JNK signaling. Apoptotic stimuli lead to loss of Cbl protein/activity, thereby removing a critical brake on JNK activation and on cell death.

Keywords: apoptosis, JNK, Cbl, MLK, NGF

Cell Research (2009) 19:950-961. doi: 10.1038/cr.2009.74; published online 23 June 2009

\section{Introduction}

There are three mammalian members of the $\mathrm{Cbl}$ (Casitas B-lineage lymphoma) family of proteins: the ubiquitously expressed c-Cbl and Cbl-b, as well as Cbl3 (also known as Cbl-c) that is expressed primarily in the aerodigestive tract. Collectively, referred to as Cbls, c-Cbl and Cbl-b have similar domains, including a modified EF Hand-containing SH2 domain known as the TKB (tyrosine kinase binding) domain, a regulatory pre-RING linker domain, a RING finger domain, a polyproline domain, and a ubiquitin-associated/leucine zipper domain [1-2]. c-Cbl and Cbl-b possess E3 ligase activity that requires an intact RING finger domain. They also exhibit the capacity for a number of different protein-protein

Correspondence: Lloyd A Greene

Fax: 212 305-5498

E-mail: lag3@columbia.edu

Received 3 November 2008; revised 15 February 2009; accepted 2 March 2009; published online 23 June 2009 interactions that appear to have functions independent of the E3 ligase activity.

c-Cbl was first identified through studies on the viral oncogene v-Cbl, a C-terminal truncation of $\mathrm{Cbl}$ that is sufficient to induce pre-B-cell lymphomas and myelogenous leukemia in mice [3]. In addition to their ability to transform cells, oncogenic forms of $\mathrm{Cbl}$ proteins such as $\mathrm{v}-\mathrm{Cbl}$ and $\mathrm{c}-\mathrm{Cbl} \Delta 371$ inhibit apoptosis induced by IL-3 withdrawal in the myeloid cell line 32Dcl13. Oncogenic Cbls also block 32Dcl13 cells from differentiating into granulocytes in response to colony-stimulating factor [45]. The mechanism for apoptosis protection by mutant Cbls seems to involve the elevation of BCL-2 by an unknown means. However, the possible roles of wild-type $\mathrm{Cbl}$ proteins in apoptosis remain less clear.

Several groups have demonstrated regulation of $\mathrm{Cbl}$ family proteins during apoptotic stress. c-Cbl/Cbl-b proteins are cleaved in Jurkat cells after exposure to UV radiation, etoposide, or anti-Fas antibodies, and this is blocked by concomitant treatment with the caspase in- 
hibitors YVAD or DEVD [6]. It has also been reported that a c-Cbl-related protein CARP (Cbl-related $90 \mathrm{KD}$ protein), a possible alternatively spliced form of c-Cbl, is upregulated in the thymus of mice treated with hydrocortisone, UV radiation, or anti-CD3 antibody [7-8]. A knock-in mouse expressing $\mathrm{c}-\mathrm{Cbl}$ with a point mutation in the RING finger domain that eliminates its E3 ligase activity has complete loss of the thymus by young adulthood. This effect can be explained by increased sensitivity to anti-CD3-induced cell death, at least in vitro [9]. In addition, a $\mathrm{T}$ helper 1 cell line derived from Cbl-b null mice had much greater sensitivity to anti-CD3-induced death than a cell line derived from Cbl-b WT mice [10]. On the other hand, c-Cbl has been shown to mediate FGFR2-mediated apoptosis of osteoblasts by downregulating $\alpha 5$ integrin and reducing cellular attachment [11]. Finally, c-Cbl knockout mice have reduced levels of apoptosis in the testis [12].

We initially became interested in Cbl proteins after identifying them as binding partners of the c-Jun Nterminal kinase (JNK) pathway scaffold POSH (plenty of SH3 domains). The JNK pathway plays a pivotal role in apoptosis of neurons and other mammalian cell types [13-15]. In particular, trophic factor deprivation models of neuronal cell death involve a sequential MAP kinase cascade including the mixed lineage kinases (MLKs), MKK 4 and 7 (mitogen-activated protein kinase kinases), and JNKs [14-20]. Once activated, JNKs then phosphorylate and activate pro-apoptotic targets such as the transcription factor c-Jun [14-15]. Initiation of the cascade appears to require activated forms of the small GTPases Cdc42 and Rac1 as well as the formation of the multiprotein POSH-JIP apoptotic complex, which includes the scaffold proteins POSH, JIPs (JNK-interacting proteins), and members of the JNK kinase cascade [17-18].

An important issue regarding the apoptotic JNKsignaling pathway is how it is suppressed in healthy cells and how it is rapidly activated in response to apoptotic stimuli. Here, we present evidence that $\mathrm{Cbl}$ proteins act as a brake on the activation of JNK signaling in healthy neuronal cells and that they are rapidly lost from cells after exposure to apoptotic conditions, thus releasing this brake. Our findings suggest that at least one mechanism for the suppression of JNK signaling by $\mathrm{Cbl}$ proteins is by inhibiting the capacity of MLKs to signal to downstream members of the JNK kinase cascade.

\section{Results}

Cbl protein levels fall in response to NGF deprivation

A previous report indicated that $\mathrm{c}-\mathrm{Cbl}$ and $\mathrm{Cbl}-\mathrm{b}$ are cleaved in Jurkat cells undergoing apoptotic stresses such as DNA damage or exposure to anti-Fas ligand [6]. Here, we assessed the state of $\mathrm{Cbl}$ proteins in cultured neuronal cells subjected to trophic factor deprivation, a treatment that triggers early JNK activation and JNK-dependent apoptotic death commencing at about 16-24 h $[15,21]$. Neuronal (nerve growth factor (NGF)-pretreated) PC12 cells (Figure 1A) and sympathetic ganglionic neurons (SGNs; Figure 1B) both showed a decrease in full-length c-Cbl expression in response to NGF deprivation. Loss of full-length c-Cbl occurred at times (4-10 h post NGF withdrawal) well before apoptotic cell death and at the time of JNK activation (Figure 1A) [21]. Cbl-b protein levels also decreased in response to NGF deprivation in both cell types (Supplementary information, Figure S1). This typically occurred at somewhat later time points than for $\mathrm{c}-\mathrm{Cbl}$, but before the time at which the majority of cells were committed to die [21-22]. We did not detect stable fragments produced by c-Cbl cleavage using an antibody directed against the C-terminal portion of this protein (data not shown).

$c$-Cbl is de-phosphorylated on tyrosine residues in response to NGF deprivation

Cbls are phosphorylated on tyrosine residues downstream of growth factor activation of receptor tyrosine kinases $[2,23]$. Of particular relevance, $\mathrm{c}-\mathrm{Cbl}$ is reported to undergo rapid phosphorylation on tyrosine residues in response to NGF [24]. Such phosphorylation activates the E3 ligase activities of Cbls and promotes their interaction with numerous proteins $[1,2,23,25]$. We therefore assessed whether NGF deprivation would result in a drop of c-Cbl tyrosine phosphorylation and if this would occur within a time frame that may be relevant to the promotion of death. To address the role of NGF specifically (that is to eliminate any effect of serum), neuronal PC12 cells were initially cultured in RPMI 1640 medium supplemented with NGF and 1\% horse serum, followed by 2 days in serum-free RPMI 1640 medium that was supplemented with NGF alone. Cells were then washed in NGF-free medium and treated with either NGF or monoclonal anti-NGF antibody in serum-free RPMI 1640 medium and lysed $3 \mathrm{~h}$ later. Immunoblot analysis of immunoprecipitated c-Cbl indicated that the levels of tyrosine-phosphorylated c-Cbl dropped significantly by $3 \mathrm{~h}$ of NGF deprivation ( $45 \pm 1.7 \%$; Figure $1 \mathrm{C})$. Thus, it appears that $\mathrm{c}-\mathrm{Cbl}$ is both rapidly degraded and rapidly de-phosphorylated in response to the apoptotic stimulus of NGF deprivation.

shRNA-mediated knockdown of Cbl/Cbl-b sensitizes neuronal PC12 cells to NGF deprivation

To mimic the loss of $\mathrm{Cbl}$ proteins and their activities 
A

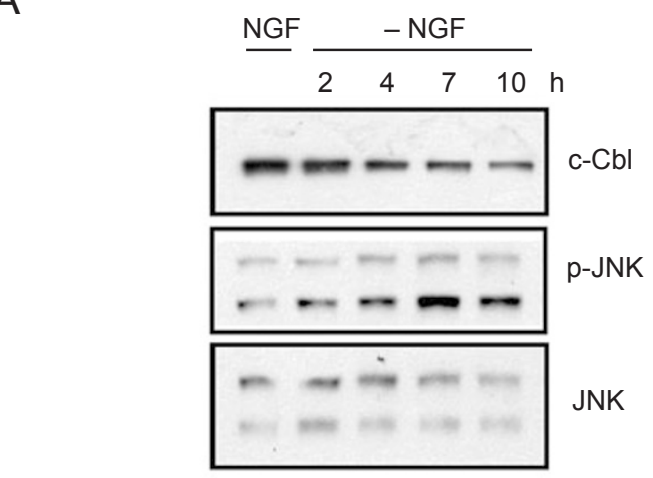

Neuronal PC12 cells

C

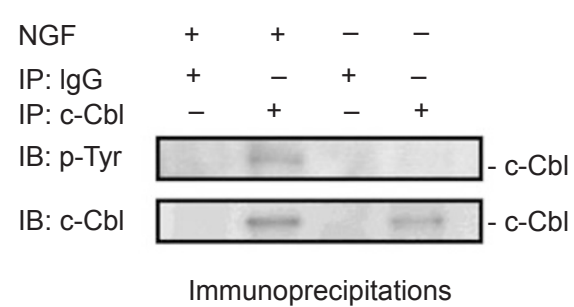

B
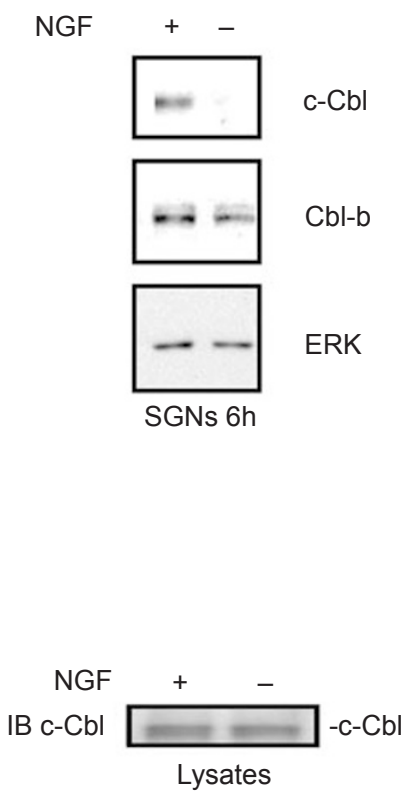

Figure 1 Cbls are regulated in response to NGF deprivation. (A-B) Neuronal PC12 cells or sympathetic ganglionic neurons (SGNs) were subjected to NGF deprivation as described in Materials and Methods. Cells were lysed directly into sample buffer and lysates were analyzed by immunoblotting. (A) Neuronal PC12 cells were analyzed for levels of c-Cbl after NGF deprivation at the time points listed, as well as p-JNK (ser63) and total JNK. (B) SGNs were analyzed by immunoblotting for $\mathrm{c}-\mathrm{Cbl}$ and $\mathrm{Cbl}-\mathrm{b}$, as well as for ERK loading control at $6 \mathrm{~h}$ post NGF deprivation. (C) Neuronal PC12 cells were grown in NGF/RPMI 1640 medium/1\% horse serum for 4 days followed by 2 days in NGF/RPMI 1640 medium without serum. Cells were washed three times and treated in RPMI 1640 medium with either NGF $(+)$ or monoclonal anti-NGF antibody $(-)$, and lysed $3 \mathrm{~h}$ later into IP buffer 2 . Aliquots of total lysate were saved for later analysis, while the rest of the NGF (+) and NGF (-) treated lysates were split in half and were subjected to immunopreciptation with either c-Cbl or control IgG antibodies. Resulting immunocomplexes and total lysate controls were subjected to immunoblotting and were probed with anti-phosphotyrosine to detect the total tyrosine phosphorylation status of c-Cbl, and then stripped and re-probed with c-Cbl antibody for loading. Total lysates were also blotted for c-Cbl levels.

caused by NGF deprivation, we designed shRNA plasmids targeting $\mathrm{c}-\mathrm{Cbl}$ or $\mathrm{Cbl}-\mathrm{b}$, utilizing sequences conserved in human, rat, and mouse. We verified the efficacy of these constructs by co-expression of target or control shRNA with HA-tagged human c-Cbl/Cbl-b constructs in HEK 293 cells (Figure 2A). Knockdown of endogenous rat c-Cbl was confirmed by immunostaining in neuronal PC12 cells (Figure 2B). We were unable to identify a commercial Cbl-b antibody that satisfactorily recognized endogenous rat $\mathrm{Cbl}-\mathrm{b}$ by immunostaining (data not shown). We first determined whether knockdown of Cbls was sufficient to induce death in the presence of trophic support (NGF). Although in one experiment there was a trend toward increased death, this was not a consistent finding $(n>6)$. In contrast, knockdown of Cbls with shRNA significantly and consistently sensitized neuronal PC12 cells to NGF deprivation (Figure 2C, D). While single knockdown of either c-Cbl or Cbl-b had some effect on cell sensitization to death, double knockdown was consistently more effective, as shown in Figure 2C. For this reason, subsequent experiments employed knockdown of both $\mathrm{Cbl}$ family members.

Cbls are regulated in camptothecin models of DNA damage and knockdown of Cbls sensitizes neuronal cells to death induced by DNA damage

To determine whether loss of Cbls was limited to NGF deprivation, we treated neuronal PC12 cells and primary embryonic cortical neurons with the DNAdamaging agent camptothecin, a topoisomerase I inhibitor that kills neurons [26]. c-Cbl and Cbl-b protein levels decreased in response to camptothecin exposure in the range of 4-11 h post treatment (Supplementary information, Figure S2A-C), again preceding cell death, which 
commences at about $18-24 \mathrm{~h}$ post treatment $[21,26]$. In addition to the loss of full-length Cbl-b, immunoblotting with an N-terminal Cbl-b antibody revealed the appearance of smaller immunoreactive species in lysates of cells subjected to NGF deprivation or camptothecin treatment (Supplementary information, Figure S2B; data not shown). This suggests that the loss of expression may be due at least in part to protein degradation. Past work has implicated caspases in the degradation of Cbls [6].
In support of this idea, the pan-caspsase inhibitor t-butoxycarbonyl-aspartyl(OMe)-fluoromethyl ketone (BAF) at least partially blocked the loss of full-length Cbl-b, as well as the formation of presumed cleavage products (Supplementary information, Figure S2B).

We also tested the effect of knocking down both Cbls in camptothecin-treated neuronal PC12 cells. In parallel with our findings for NGF deprivation, cells transfected with shRNAs targeting Cbls were significantly sensitized
A

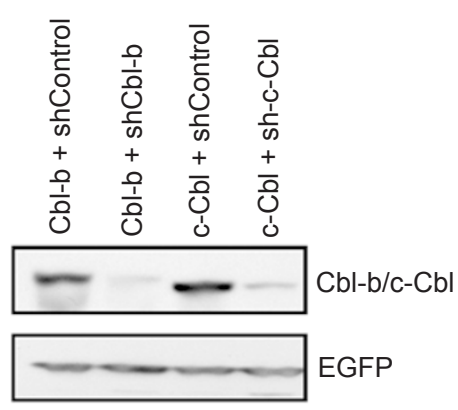

C

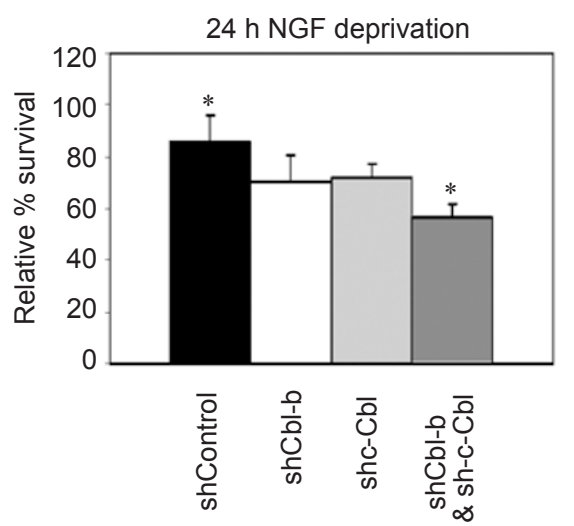

B
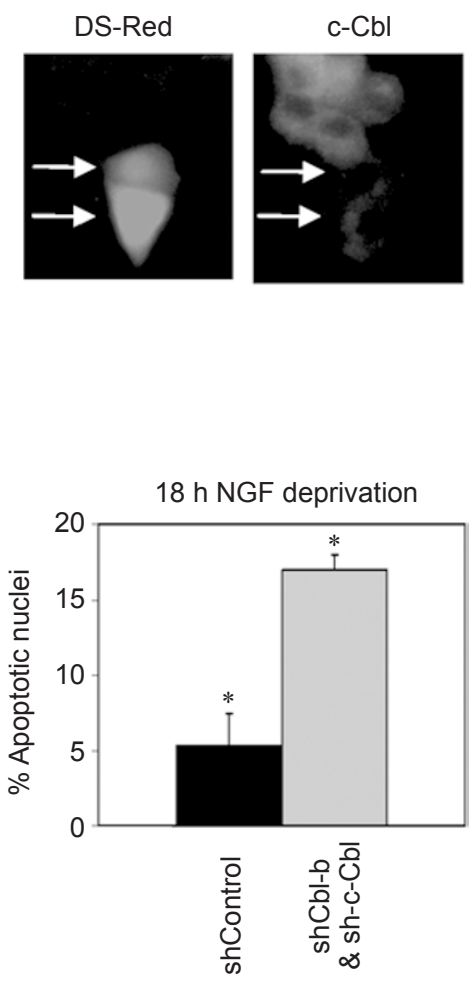

Figure 2 shRNA targeting Cbls sensitizes neuronal PC12 cells to NGF deprivation. (A) 293 cells were co-transfected with shRNA, either control shLuc (shControl), shCbl-b, or sh-c-Cbl in the pSiren vector background, in a 4:1 ratio with either c-Cbl or Cbl-b in a bicistronic pCMS.EGFP vector. Cells were lysed $48 \mathrm{~h}$ later directly into sample buffer, and analyzed for levels of $\mathrm{Cbl}$ proteins using the G1 Cbl-b antibody, which detects both overexpressed proteins. EGFP was used as a transfection loading control. (B) Neuronal PC12 cells were transfected with sh-c-Cbl, fixed $48 \mathrm{~h}$ later, and immunostained for levels of c-Cbl using the C-15 c-Cbl antibody (right panel). Transfected cells (DsRed positive) are indicted by arrows. (C-D) Neuronal PC12 cells were transfected with control shLuc EGFP and shLuc DsRed (shControl), shCbl-b EGFP (balanced with shLuc DsRed), sh-c-Cbl DsRed (balanced with shLuc EGFP) or shCbl-b EGFP and sh-c-Cbl DsRed. After 2 days, transfected cells were washed and maintained with NGF or without NGF and with anti-NGF antiserum as indicated. (C) Survival was measured by counting the same vertical strip of transfected cells immediately after NGF deprivation (zero time) and $24 \mathrm{~h}$ later. At least three wells were assessed for each condition, and one representative experiment of at least three independent experiments (each with comparable results) is shown. Counts for each well culture were normalized to the zero time and then compared as indicated for survival relative to that in cultures maintained with NGF under the same conditions of transfection. Values are means \pm SEM $(n=3)$. $* P<0.05$, Student's $t$-test. (D) At $18 \mathrm{~h}$ following NGF deprivation, transfected cells were stained with Hoechst dye 33342 and scored for percentage of nuclei with apoptotic morphology. At least 50 nuclei were scored under each condition. A representative experiment is shown. Comparable results were achieved in a total of three independent experiments. ${ }^{*} P<0.05$, Student's $t$-test. 
to camptothecin treatment as compared to those transfected with control shRNAs (Supplementary information, Figure S2D).

\section{Cbls are regulated by acute exposure to NMDA}

To address whether $\mathrm{Cbl}$ levels might be regulated in additional paradigms of neuronal death, we examined an acute NMDA model of death in cultured cortical neurons [27]. Both Cbl-b (Supplementary information, Figure S2E) and c-Cbl (data not shown) were rapidly decreased in response to NMDA treatment. This effect was significantly blocked by the calpain inhibitor calpeptin, thus indicating a mechanism at least in part dependent on degradation. The formation of presumed cleavage fragments, which were also blocked by calpeptin, still occurred in the presence of Actinomycin D (data not shown), which argues that they are not derived from novel transcripts.

\section{Cbl proteins interact with JNK pathway components}

We initially found an association between Cbl proteins and the JNK pathway from a yeast-two hybrid screen that used a full-length construct of the JNK scaffold POSH as bait $[28,29]$. This screen identified both c-Cbl and Cbl-b (3/52 hits) as medium/strong binding partners of full-length POSH. We first verified this interaction in HEK293 cells by co-expressing epitope-tagged $\triangle \mathrm{ZnPOSH}$ (a RING finger domain deletion mutant that is more stable and thus more highly expressed than $\mathrm{wt}$ POSH [17]) and c-Cbl/Cbl-b. Myc- $\triangle \mathrm{ZnPOSH}$ and HA$\mathrm{c}-\mathrm{Cbl} / \mathrm{Cbl}-\mathrm{b}$ were co-immunoprecipitated in both directions and this interaction did not require POSH's RING finger domain (Supplementary information, Figure S3). To investigate whether the POSH and $\mathrm{Cbl}$ protein interaction is direct, we performed GST-pulldowns with $\mathrm{N}$-terminal and C-terminal halves of GST-POSH and in vitro transcribed/translated c-Cbl/Cbl-b (Supplementary information, Figure S3). Both halves of POSH pulled down full-length c-Cbl, Cbl-b, and Cbl-b 2/3 (a mutant missing the $\mathrm{N}$-terminal TKB domain). Despite these findings with overexpressed POSH and Cbls, we were unable to demonstrate interaction of the endogenous proteins in multiple co-IP experiments using extracts from both neuronal and non-neuronal cells with or without the use of proteasome inhibitors (data not shown). This suggested either that complexes formed by these proteins were at levels or transitory stabilities below our capacity to detect or that the interaction does not occur under more physiological conditions. Finally, overexpression of either wild-type c-Cbl or the dominant-negative $\mathrm{v}-\mathrm{Cbl}$ did not appear to affect endogenous POSH levels in 293 cells (Supplementary information, Figure S4).

In addition to POSH, MLKs appear to be limiting
A

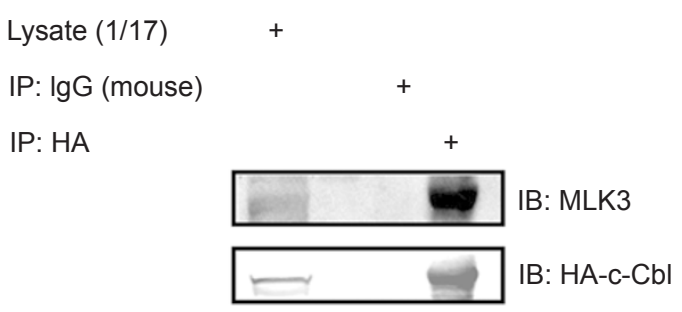

B

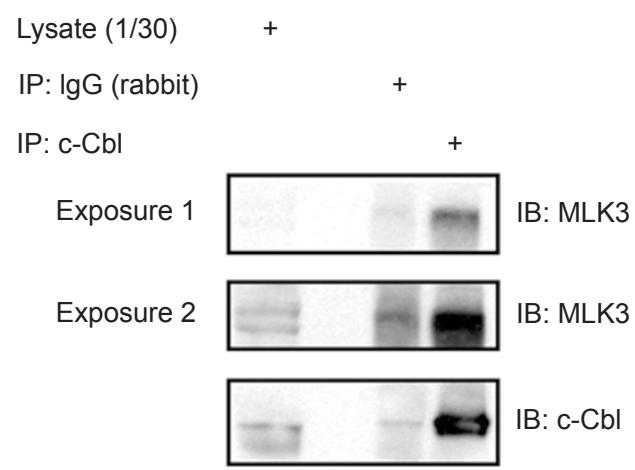

Figure $3 \mathrm{c}-\mathrm{Cbl}$ Interacts with endogenous MLK3. The fraction listed for each lysate is the ratio of the volume of lysate versus the total lysate volume used in each immunoprecipitation. Each panel shows a representative experiment of two independent experiments. (A) HEK 293 cells were transfected with $\mathrm{HA}-\mathrm{C}-\mathrm{Cbl}$ and lysed $26 \mathrm{~h}$ later into IP buffer \#1. Aliquots of total lysate were saved for later analysis, while the rest was split in half and subjected to immunoprecipitation with either IgG (mouse) alone or monoclonal anti-HA antibodies, followed by western blot analysis of MLK3 and HA-c-Cbl. (B) PC12 cells were treated with the proteasome inhibitor Mg132 for $6 \mathrm{~h}$ and then were lysed into IP buffer \#1. Aliquots of total lysate were saved for later analysis, while the rest was split in half and subjected to immunoprecipitation with either lgG (rabbit) alone or c-Cbl (C-15) antibody, followed by western blot analysis of MLK3 and c-Cbl. Comparable results were found in one experiment with PC12 cell cultures in which no MG132 was added (data not shown).

components of the JNK signaling cascade and inhibition of MLK activity protects neurons from death induced by NGF deprivation or DNA damage $[16,20]$. We therefore assessed whether $\mathrm{c}-\mathrm{Cbl}$ and the MLK family member MLK3 interact. As shown in Figure 3A, overexpressed HA-tagged c-Cbl co-immunoprecipitated with endogenous MLK3 in lysates of 293 cells. In the converse direction, overexpressed tagged MLK3 was able to co-immunoprecipitate tagged c-Cbl (data not shown). In addition, endogenous c-Cbl specifically co-immunoprecipitated with endogenous MLK3 in PC12 cells treated with the proteasome inhibitor MG132 (Figure 3B). Similar findings were achieved in cells not exposed to MG132 (data 
A

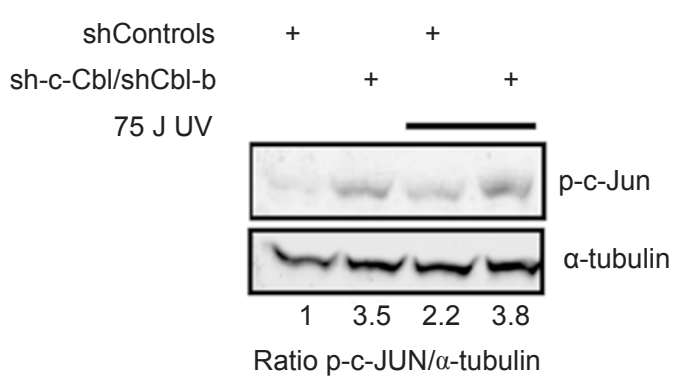

B

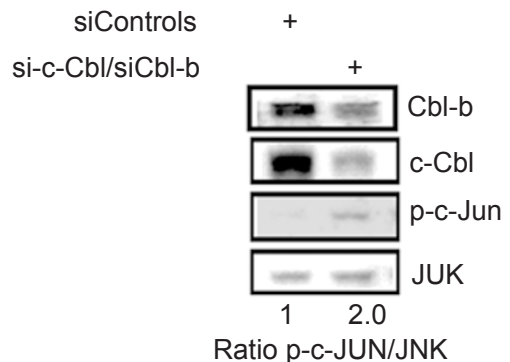

Figure 4 RNAi targeting Cbls activates JNK signaling. (A) HEK 293 cells were co-transfected with either shLuc EGFP and shLuc DsRed (shControls), or shCbl-b EGFP and sh-c-Cbl DsRed in duplicate wells. One well from each set of co-transfections was exposed to $75 \mathrm{~J}$ UV. All cells were lysed $2 \mathrm{~h}$ later directly into sample buffer, and analyzed by immunoblotting for levels of phospho-c-Jun (ser63) and $\alpha$-tubulin as a loading control. The ratio of p-c-Jun to $\alpha$-tubulin was analyzed for each using Odyssey software, and normalized to shLuc controls in unstressed cells. (B) HEK 293 cells were co-transfected with either two different siControl oligos (siControl A\&B) or si-c-Cbl and siCbl-b, and lysed $48 \mathrm{~h}$ later directly into sample buffer. Samples were analyzed by immunoblotting for $\mathrm{p}$-c-Jun and JNK, and normalized relative to siControl transfected cells utilizing Image J.

not shown). The properties of existing MLK3 antisera precluded carrying out the converse co-immunoprecipitation of the endogenous proteins. Overall these findings support the idea that $\mathrm{c}-\mathrm{Cbl}$ specifically interacts with MLK3 in living cells.

\section{Reduction of Cbls via RNAi activates the JNK pathway}

Neuronal apoptosis induced by both NGF deprivation and DNA damage is associated with prolonged, moderate (2-3 fold) activation of the JNK-signaling pathway and this activity is required for death [14-16, 21]. Furthermore, our data indicate that $\mathrm{Cbl}$ proteins interact with JNK pathway components and are downregulated/inactivated in response to apoptotic stimuli during time periods that correlate with JNK pathway activation. We therefore assessed the possibility that loss/inactivation of $\mathrm{Cbl}$ proteins contributes to the activation of JNK signaling. We utilized HEK 293 cells due to their high transfection efficiencies (in contrast to neuronal PC12 cells and sympathetic neurons, which have transfection efficiencies on the order of $1 \%$ or less) to examine the effect of $\mathrm{Cbl}$ knockdown on JNK pathway activity. Compared with cells transfected with two control shRNAs, knockdown of both c-Cbl and Cbl-b in HEK 293 cells led to a threefold increase in activation of the JNK-signaling pathway, as indicated by phosphorylation of its target c-Jun at Ser63 (Figure 4A). UV irradiation was used as a positive control for JNK pathway activation. We confirmed these results by utilizing commercially available siRNAs targeting human c-Cbl and Cbl-b (Santa Cruz pool of three siRNAs per target), which have targeting sequences different from the shRNA sequences we employed (Figure 4B). These findings indicate that loss of Cbls leads to activation of JNK signaling and support the hypothesis that, when present, Cbls provide a brake on JNK signaling.

c-Cbl proteins block MLK signaling in a RING-independent manner

We next investigated the possible mechanisms by which Cbls might suppress JNK signaling in healthy cells. MLKs and POSH, with which Cbls interact, are maintained at low levels in healthy cells and are stabilized in response to apoptotic stimuli, thereby reaching sufficient levels to promote JNK pathway activation and cell death $[17,19]$. Because Cbls are E3 ligases, we first investigated whether they might, in healthy cells, promote degradation of POSH whose stability is proteasome-dependent [17]. Overexpressed wild-type c-Cbl or Cbl-b had little to no effect on endogenous POSH expression in HEK293 cells (Supplementary information, Figure S4 and data not shown). Thus it appears that at least under the conditions of our study, Cbls do not target POSH for degradation.

We next assessed whether Cbls might suppress JNK signaling by reducing MLK levels. Unexpectedly, endogenous MLK3 was greatly stabilized by expression of wild-type or RING finger mutant c-Cbl (C381A) as shown in Figure 5A, B. Furthermore, this stabilization also led to elevated levels of phosphorylated (and presumably activated) MLK3 (Figure $5 \mathrm{C}$ ). This effect did not occur with $\mathrm{Cbl}-\mathrm{b}$ or $\mathrm{v}-\mathrm{Cbl}$, which appears to act as a dominant-negative version for at least some c-Cbl functions including E3-mediated degradation of receptor tyrosine kinases [2, 3] (Figure 5B, C and data not shown). Because Cbls did not destabilize MLKs, we 
next considered the possibility that they might interfere with their ability to promote JNK signaling. As shown in Figures 5A-5C, despite the large increase in level and activation of MLK3 that occurs in the presence of overexpressed $\mathrm{c}-\mathrm{Cbl}$, this does not result in enhanced phosphorylation of MKK4, JNKs, or of the JNK substrate c-Jun. As one control for JNK pathway activation, we overexpressed MLK3 at a relatively low level, and even though total cellular levels of this protein did not reach those achieved by overexpression of $\mathrm{c}-\mathrm{Cbl}$, there was robust JNK phosphorylation (Figure 5A). As another positive control for JNK pathway activation, parallel transfected HEK 293 cells were treated with UV irradiation. In the UV irradiation model of cell death in this cell type, JNK pathway activation appears to be mediated by kinases other than MLKs [30,31]. UV irradiation led to increased phosphorylation of both MKK4 and c-Jun, and in line with the reported MLK-independent mechanism, this was not affected by c-Cbl overexpression (Figure $5 \mathrm{~B}, \mathrm{C})$. These findings thus support the possibility that c-Cbl interferes with the capacity of activated MLKs to signal to downstream components of the JNK cascade, including MKK4, the next component in the cascade.

Overexpression of $\mathrm{c}$-Cbl protects neuronal cells against $M L K$ overexpression in a RING-independent manner

Expression of MLKs promotes JNK-dependent apoptosis in neuronal PC12 cells [16]. If c-Cbl can block the ability of an MLK to activate JNK signaling, it follows that $\mathrm{c}-\mathrm{Cbl}$ should interfere with the capacity of MLKs to promote cell death. We tested this hypothesis by cotransfecting neuronal PC12 cells with MLK3 or the related family member MLK2 and either empty vector or various c-Cbl constructs, and measuring apoptotic death. We used both wild-type c-Cbl and a reportedly constitutively active E3 ligase version, c-Cbl371E [25], that has the Y371 activation residue mutated to glutamate to mimic phosphorylation. Expression of either wild type or c-Cbl371E protected the cells against apoptosis induced by MLK overexpression (Figure 6A, B). We also assessed whether Cbl RING finger activity is required for c-Cbl to block MLK-induced death. As shown in Figure 6A, RING finger mutant c-Cbl-381A also effectively protected cells from MLK3 overexpression. Thus, E3 ligase activity does not appear to be necessary for the capacity of c-Cbl to block MLK death signaling.

Because our findings indicate that c-Cbl blocks JNK signaling at the level of MLKs, we next assessed the capacity of c-Cbl371E to inhibit cell death promoted by a MAPKK downstream of MLKs, MKK7. In this case, there was no suppression of death (Figure 6C). Similar results were found in one experiment testing the ability
A

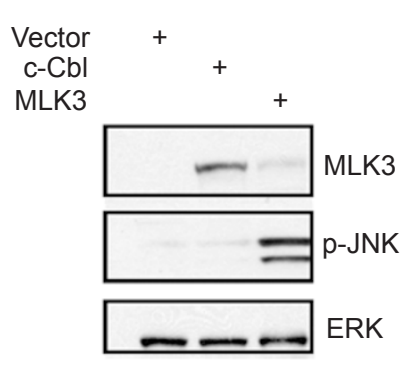

B

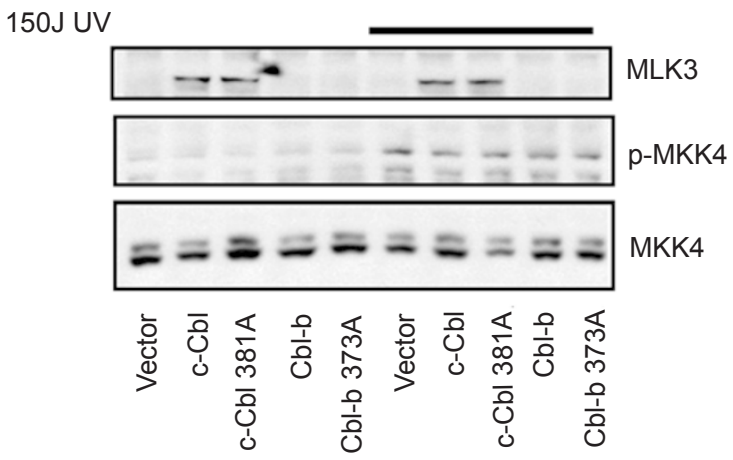

C

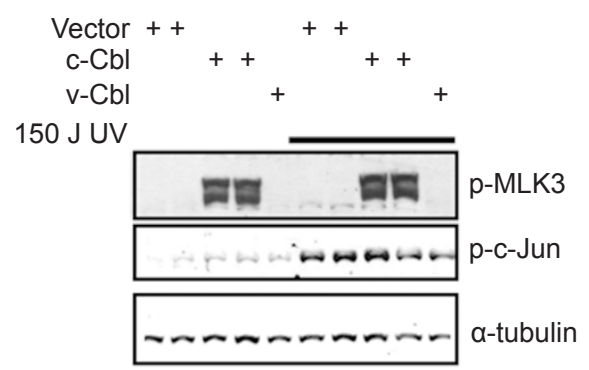

Figure 5 Effects of Cbls on MLK3 levels and activity. (A-C) EGFP vector, Cbl constructs, and Flag-MLK3 were transfected alone and in combination into 293 cells, lysed the next day directly into sample buffer, and analyzed by immunoblot as indicated. For (B) and (C), parallel transfected cultures were subjected to $150 \mathrm{~J}$ UV (lysed $2 \mathrm{~h}$ post treatment) to use as a positive control for JNK pathway activation. (A) Lysates were analyzed for total MLK3 and phosphorylated JNKs. (B) Lysates were analyzed for endogenous phosphorylated MLK3, phosphorylated c-Jun, and $\alpha$-tubulin. (C) Lysates were analyzed for total endogenous MLK3, phosphorylated MKK4, and total MKK4.

of MKK4 to kill neuronal PC12 cells (data not shown). This is consistent with the model that $\mathrm{c}-\mathrm{Cbl}$ suppresses the JNK-signaling cascade between MLKs and MKK4/7 and not downstream of MKK4/7 activation. Finally, we tested whether co-expression of Cbl-b protects against overexpression of MLK3 in neuronal PC12 cells and 
A

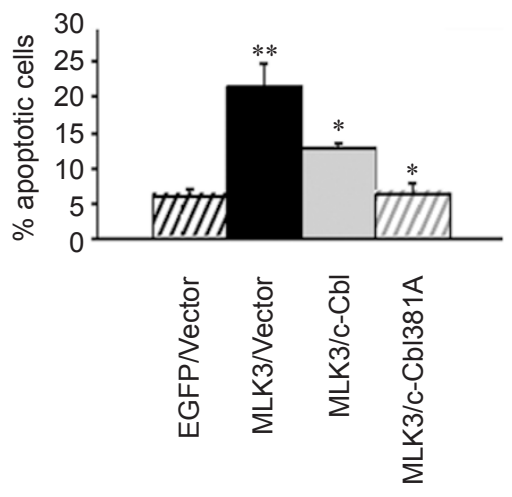

C

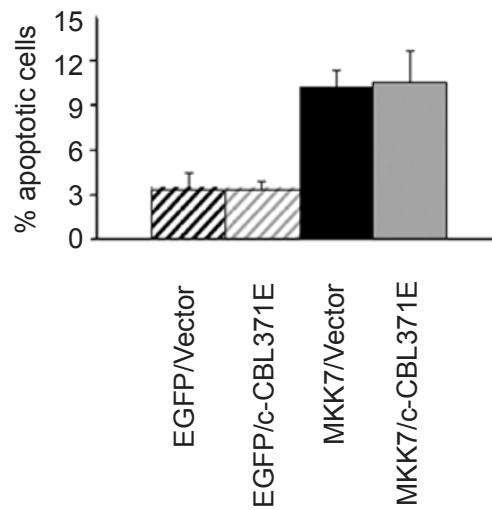

$\mathrm{B}$

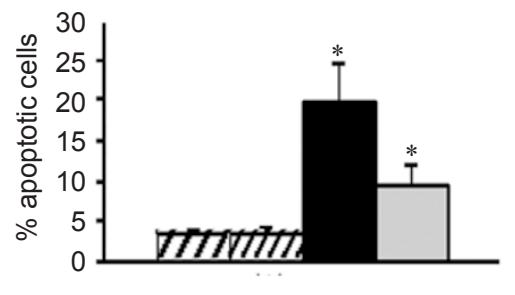

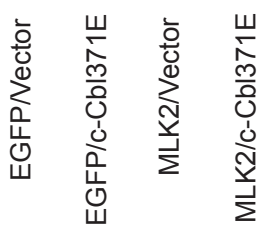

D

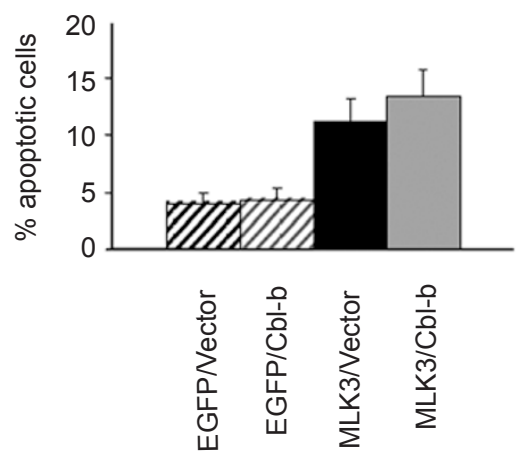

E

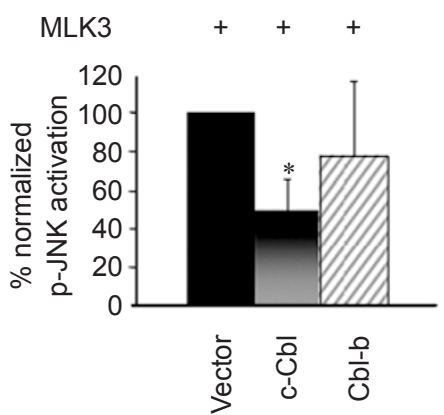

Figure $6 \mathrm{c}$-Cbl blocks MLK, but not MKK-mediated cell death. (A-D) Neuronal PC12 cells were transfected as indicated, fixed $24 \mathrm{~h}$ later, and blindly scored for apoptotic morphology as in Figure 2 ( $n=3$ for each condition). (A) Cells were co-transfected with either MLK3 or EGFP and either empty pCefl vector, c-Cbl, or c-Cbl 381A. One representative experiment is shown of two independent experiments. ${ }^{*}$ is statistically different from both $*$ conditions, $P<0.05$, Student's $t$-test. (B) Cells were cotransfected with either EGFP or EGFP.MLK2, and with either empty pCefl vector or c-Cbl 371E. One representative experiment of three independent experiments is shown. ${ }^{*} P<0.05$, Student's $t$-test. (C) Cells were co-transfected with either EGFP or EGFP.MKK7, and with either empty pCefl vector or c-Cbl 371E. Similar results were obtained by utilizing EGFP.MKK4 instead of EGFP.MKK7 (data not shown). (D) Cells were co-transfected with either MLK3 or empty vector, and with either empty EGFP vector or with EGFP.Cbl-b. One representative experiment of two independent experiments is shown. (E) Either empty EGFP vector or Flag-MLK3 was co-transfected with either empty pCefl vector, c-Cbl, or Cbl-b into 293 cells. The next day, cells were lysed directly into sample buffer, and analyzed by immunoblotting for Flag-MLK3 levels, p-JNK, and total JNK levels. The graph shows the quantification of pJNK to JNK ratios for four bands for each condition (total two experiments), normalized to relative MLK3 levels. Vector was defined as 100\%. * indicates c-Cbl at $95 \%$ confidence did not overlap the vector $100 \%$ value (alpha $=17 \%)$. 
found that in contrast to c-Cbl, it does not (Figure 6D).

To determine whether the capacity of $\mathrm{c}-\mathrm{Cbl}$ to protect cells from MLK3-promoted death correlates with its ability to inhibit JNK signaling, we co-transfected HEK293 cells with MLK3 in the presence or absence of c-Cbl or Cbl-b and assessed JNK phosphorylation. As shown in Figure 6E, c-Cbl significantly diminished JNK activation, while there was no significant effect of Cbl-b.

\section{Discussion}

Given that a previous report indicated that $\mathrm{Cbl}$ protein levels fall during apoptotic stress in Jurkat cells [6] and our interest in potential effectors of neuronal cell death, we examined whether Cbl levels also fall in neuronal death models. The levels of $\mathrm{c}-\mathrm{Cbl}$ and $\mathrm{Cbl}-\mathrm{b}$ proteins were both reduced in response to NGF deprivation and camptothecin treatment. In addition to being cleaved in response to NGF deprivation, c-Cbl also underwent rapid loss of tyrosine phosphorylation. Mutational studies suggest that phosphorylation at tyrosine 371 of $\mathrm{c}-\mathrm{Cbl}$ promotes its E3 ligase activity, whereas deletion of this residue is oncogenic $[25,32]$. In addition, phosphorylation at three $\mathrm{C}$-terminal tyrosine residues promotes the interaction of $\mathrm{c}-\mathrm{Cbl}$ with a variety of proteins, such as Vav, Crk proteins, and the p85 subunit of PI3K [1]. One would therefore predict that decreased tyrosine phosphorylation should diminish c-Cbl's E3 ligase activity as well as its binding to many partners. Taken together, our findings indicate that apoptotic stimuli lead to a rapid loss of $\mathrm{c}-\mathrm{Cbl}$ and $\mathrm{Cbl}-\mathrm{b}$ protein levels in neuronal cells and a potential loss of c-Cbl activity, and that these effects occur well before the onset of cell death.

To examine the significance of reduced $\mathrm{Cbl}$ proteins and their activities, we mimicked this effect utilizing shRNAs targeting Cbls. Knockdown of Cbls, while not sufficient to promote significant death of neuronal PC12 cells on its own, sensitized them to NGF withdrawal and camptothecin treatment. In a number of cases, such as for EGF, PDGF, and SCF receptors among others, Cbls act as E3 ligases to promote receptor degradation [1, 2, $23,33]$. In these instances, loss of Cbls or of Cbl activity would be expected to strengthen survival signaling by increasing the number of receptors at the cell surface. However, TrkA, the receptor for NGF, phosphorylates (and presumably activates) c-Cbl without being downregulated in turn ([34]; Sproul A, Greene L, unpublished observations). Thus, neurons that do not compete well for limited supplies of NGF during development would undergo loss of $\mathrm{Cbl}$ protein and activity without a compensatory increase in Trk signaling and thus undergo a more efficient death process.
It is likely that this sensitization to cell death reflects effects of Cbl knockdown on multiple signaling pathways, as c-Cbl has been found to interact with over 150 proteins [1]. We focused here on the JNK pathway, a critical mediator of neuron death activated by NGF withdrawal and other apoptotic stimuli [13-15]. We observed that $\mathrm{Cbl}$ can interact with two JNK pathway components, MLK3 and POSH. Moreover, loss of Cbl proteins correlates temporally with the two- to three-fold activation of the JNK pathway that occurs in response to NGF deprivation. In support of a role for Cbls in regulation of JNK signaling, knockdown of Cbls in HEK 293 cells (a system in which transfection efficiencies are high enough to carry out biochemical studies) was sufficient to promote JNK activation by two- to three-fold. Recent findings indicate that physiological activation of JNK signaling is necessary, but not sufficient to trigger neuronal death [35]. Such observations may explain why loss of Cbls, although sufficient to activate JNK signaling, sensitizes neuronal cells to death but does not trigger apoptosis on its own.

An additional goal of our study was to determine how Cbls might suppress JNK signaling in healthy cells and how loss/inactivation of $\mathrm{Cbl}$ proteins in response to apoptotic stimuli could trigger activation of JNK signaling. An obvious mechanism by which Cbls might block JNK signaling would be via E3 ligase-dependent turnover of POSH or MLKs whose levels are low in healthy neuronal cells and rise in response to apoptotic stimuli $[16,17,19,20]$. However, this was not the case and unexpectedly c-Cbl, but not Cbl-b, stabilized endogenous and overexpressed MLK3 and elevated its phosphorylation levels at autophosphorylation sites that are critical for MLK3 activity [36]. Nevertheless, while overexpressed c-Cbl stabilized "activated" MLK3, it suppressed its capacity to promote phosphorylation and activation of c-Jun, JNK, and MKK4/7, as well as to induce neuronal cell death. Thus, c-Cbl appears to suppress JNK signaling after MLK activation, and this brake is released in response to apoptotic stress (Figure 7, model). It is probable that there are additional mechanisms by which Cbls inhibit JNK activation, particularly for Cbl-b, which in contrast to c-Cbl, does not stabilize MLK3 or block MLK3-induced apoptosis or JNK activation. For example, Cbl-b (as well as c-Cbl) has been reported to inhibit the ability of the GEF Vav to activate JNKs [37, 38].

The mechanism by which c-Cbl suppresses signaling between MLKs and MKKs is presently unclear. Our findings indicate that c-Cbl's E3 ligase activity is not required for this effect and therefore that it likely involves a non-catalytic protein-protein interaction. Preliminary evidence suggests that $\mathrm{c}-\mathrm{Cbl}$ does not interfere with the 
A

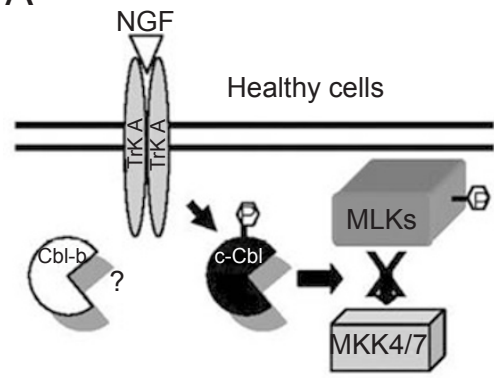

B

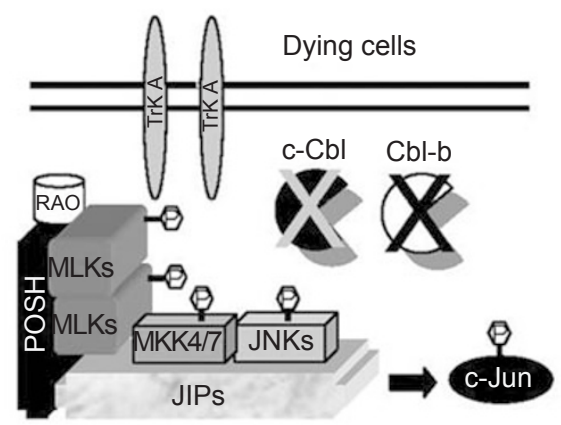

Figure 7 Model of $\mathrm{Cbl}$ action. In healthy neuronal cells (A) phosphorylated c-Cbl is at sufficient levels to block the transmission from MLKs to MKK4/7. In contrast, in dying neuronal cells such as those where NGF has been withdrawn (B) c-Cbl protein levels drop, thus releasing inhibition of MLK signaling in the POSH-JIP apoptotic complex (PJAC). Although Cbl-b levels are also regulated in response to apoptotic stress, the mechanism of how they contribute to cell death is currently unclear.

ability of MLKs to bind POSH, or with POSH's ability to bind JIPs (Sproul A, unpublished findings). An alternative possibility is raised by the observations that MLKs, and in particular MLK3, need to oligomerize to propagate their signal to JNKs [39-41]. For instance, a mutation in MLK3 that prevents oligomerization, but still allows its activation by $\mathrm{Cdc} 42$ prevents it from activating JNKs. Our findings that MLK3 and c-Cbl can be isolated within the same complex raises the possibility that such an interaction leads to inhibition of MLK signaling, perhaps by interfering with their oligomerization.

In summary, our findings support a model in which Cbls suppress apoptotic JNK signaling in healthy neurons. This occurs at least in part by interference with the capacity of activated MLKs to signal to downstream components in the JNK-signaling cascade. In response to apoptotic stimuli such as NGF deprivation and DNA damage, Cbls levels rapidly fall, relieving their inhibition of apoptotic JNK signaling.

Cbls and MLKs are widely distributed. Although we have focused on a neuronal context, it is likely that the mechanism proposed here also pertains to MLKdependent death in other cell types. Moreover, inhibition of MLK/JNK signaling by Cbls may be important in additional contexts in which these kinases regulate cellular behavior.

\section{Materials and Methods}

\section{Antibodies}

Anti c-Cbl (C-15), Cbl-b (G1), ERK, Myc, and control IgGs from rabbit and mouse were purchased from Santa Cruz (Santa Cruz, CA, USA). Anti-phospho MLK3 (Thr 277/Ser281), MLK3, phospho JNK (Thr183/Tyr185), JNK, phospho MKK4 (Ser257/
Thr261), MKK4, phospho MKK7 (Ser271/Thr275), MKK7, phospho c-Jun (Ser63 and Ser73), and c-Jun antibodies were from Cell Signaling (Beverly, MA, USA). Anti-NGF, FLAG, HA (rabbit), PY20 (phospho-tyrosine) antibodies were from Sigma (St Louis, MO, USA). Anti-POSH antibody was from Abnova (Taiwan). Anti-HA (mouse) was from Covance (Berkeley, CA, USA).

\section{Chemicals and reagents}

Anti-human recombinant NGF, camptothecin, anti-c-Myc agarose conjugate, and Hoechst dye 33342 were from Sigma. Protein A Sepharose 4 Fast Flow beads and ECL were from GE Healthcare (Piscataway, NJ, USA). Super Signal West Dura Extended Duration Substrate and mouse and rabbit secondary HRP-conjugated antibodies were from Pierce (Rockford, IL, USA). NuPAGE BisTris Protein Gels, Lipofectamine 2000, and secondary antibodies for immunofluorescence studies (Molecular Probes), were from Invitrogen (Carlsbad, CA, USA). Anti-mouse IgG conjugated to IRDye 800 was purchased from Rockland (Gilbertsville, PA, USA). BAF was purchased from Enzyme Systems Products (Livermore, CA, USA) and calpeptin from Calbiochem (EMD, Gibbstown, NJ, USA).

Cell culture, transfections, and assessment of cell death

Naïve PC12 cells were grown on collagen-coated plates in RPMI 1640 medium supplemented with $10 \%$ horse serum and 5\% fetal bovine serum. Neuronally differentiated PC12 and dissociated rat superior cervical SGNs were grown in RPMI 1640 medium supplemented with recombinant human NGF (50 ng/ml, kindly provided by Genetech Inc.) and $1 \%$ horse serum as described previously [16]. Cortical rat neurons used for camptothecin studies were dissected at age E19, plated on polylysine, and grown in DMEM/F12 medium supplemented as described previously [19]. E19 Embryonic cortical neurons utilized for NMDA experiments were grown in Neurobasal medium with the addition of B27 Supplement without antioxidants (Invitrogen) for 13 days postplating before acute 20 minutes treatment with $300 \mu \mathrm{M}$ NMDA or mock treatment in Earl's basal salts as has been done previously, with the change of re-adding conditioned media post treatment [27]. 
PC12 and primary neurons were transfected with Lipofectamine 2000 as per the manufacturer's instructions (Invitrogen). HEK 293 cells were cultured in plastic culture dishes and grown in DMEM supplemented with $10 \%$ fetal bovine serum, and transfected as transcribed previously [16], with Expressfect as per the manufacturer's instruction (Denville Scientific, Metuchen, NJ, USA), or with RNAimax as per the manufacturer's instructions (Invitrogen). All survival (by strip counting) or death assays (apoptotic nuclear morphology) were scored blindly as described previously [16].

\section{Plasmids and RNAi}

Myc-POSH, Myc- $\triangle \mathrm{ZnPOSH}$, GST-POSH N and C-terminus, Flag-MLK3, HA-MLK2, MKK4, and MKK7 constructs have been described previously [16, 17].

pCefl.HA-c-Cbl, pCefl.HA-c-Cbl C381A, pCefl.HA-Cbl-b, pCefl.Cbl-b C373A, pCefl.Cbl-b C 2/3, and empty pCefl vector were generously given by Dr Stanley Lipkowitz (National Cancer Institute, MD, USA). pCMS.EGFP was cut with EcoR1 and blunted with Klenow to sub-clone $\mathrm{Cbl}$ constructs. pCMS.EGFP.c-Cbl and $\mathrm{c}-\mathrm{Cbl} \mathrm{C} 381 \mathrm{~A}$ were generated by excision of $\mathrm{c}-\mathrm{Cbl}$ from the pCefl vector utilizing BamH1, blunting with Klenow , and ligating into blunted pCMS.EGFP. pCMS.EGFP.Cbl-b, Cbl-b C373A, and Cbl-b 2/3 were generated by excision of Cbl-b from the pCefl vector utilizing HindIII and Kpn1, blunting with Klenow, and ligating into blunted pCMS.EGFP. pCMS.EGFP.Flag-v-Cbl was constructed by PCR amplification of pCMS.EGFP.HA-c-Cbl utilizing the following primers, cutting the amplicon with $X h o 1 / X b a 1$ and cloning into similarly cut $\mathrm{pC}$ efl vector.

5' CTC GAG AAC CAT GGA CTA CAA GGA CGATGA TGA CAA AGC CGG CAA CGT GAA GAA GAG C.

3' TGT GTC TAG ATC AGG GAG TTG GTT CAC ATA AGC CAG.

pCefl.c-Cbl 371E (constitutive active) was constructed utilizing the QuikChange sited directed mutagenesis kit (Stratagene), using the following primers:

5' GTG ACC CAG GAA CAA TAT GAA TTA GAG TGT GAG ATG GGC TCC ACA TTC C

3' GGA ATG TGG AGC CCA TCT CAC ACT CTA ATT CAT ATT GTT CCT GGG TCA C

shRNA targeting c-Cbl and Cbl-b were constructed by using pSiren vector kits (pSiren and pSiren-DsRed Xpress for both constructs) as per the manufacturer's instructions, utilizing their shLuciferase (shControl) primers as well as the following (target sense sequence underlined):

$\mathrm{c}-\mathrm{Cbl}$

Sense GAT CCG ACG GTG GAC AAG AAG ATG GTG GTT CAA GAG ACC ACC ATC TTC TTG TCC ACC GTT TTT TTG G

Antisense AAT TCC AAA AAA ACG GTG GAC AAG AAG ATG GTG GTC TCT TGA ACC ACC ATC TTC TTG TCC

Cbl-b

Sense_GAT CCG ATC TTC AGT CAC ATG CTG GCA GTT CAA GAG ACT GCC AGC ATG TGA CTG AAG ATT TTT TTG G

Antisense AAT TCC AAA AAA ATC TTC AGT CAC ATG CTG GCA GTC TCT TGA ACT GCC AGC ATG TGA CTG AAG ATG G

shLuciferase EGFP (shControl EGFP) and shCbl-b EGFP were constructed by excising the shRNA sequence and upstream U6 promoter out of the pSiren vector via $b g I 1$ and EcoR 1 , followed by ligation into similarly cut pCMS.EGFP (which lost the CMV promoter region but retained the EGFP cistron).

siRNA oligos were purchased from Invitrogen, and include siCbl h2 (sc-44254), siCbl-b h, (sc-29950), control siRNA-A (sc37007), and control siRNA-B (sc-44230).

\section{Co-Immunoprecipitation, immunoblotting and in vitro bind- ing assays}

Co-immunoprecipitation and immunoblotting and in vitro binding assays were performed as previously described except as noted below $[16,17]$. pCefl.c-Cbl, $p C e f l . C b l-b$, and $p C e f l . C b l-b$ $2 / 3$ was used for in vitro transcription and translation using the TNT-coupled reticulocyte lysate system (Promega, Madison, WI, USA). IP buffer \#1 was as described previously [17]. IP Buffer \#2 was used for immunoprecipitation of $\mathrm{c}-\mathrm{Cbl}$ for phospho-tyrosine analysis, and is composed of the following made in $1 \mathrm{x}$ TBS final: $1 \%$ Triton X, 0.5\% Deoxycholate, $0.1 \%$ SDS, $1 \mathrm{mM}$ EDTA, $1 \mathrm{mM}$ Vanadate, and protease inhibitor tablet (Roche).

\section{Acknowledgments}

This work was supported by grants from the NIH/NINDS (NS33689) (L.A.G.) and from the National Science Foundation of China (NSFC) (30525007/30670663), the Ministry of Science and Technology of China (2006AA02Z173/2007CB947202) and the Chinese Academy of Sciences (KSCX1-YW-R-59) (Z.X.).

\section{References}

1 Schmidt $\mathrm{MH}$, Dikic I. The Cbl interactome and its functions. Nat Rev Mol Cell Biol 2005; 6:907-919.

2 Swaminathan $\mathrm{G}$, Tsygankov $\mathrm{AY}$. The $\mathrm{Cbl}$ family proteins: ring leaders in regulation of cell signaling. J Cell Physiol 2006; 209:21-43.

3 Langdon WY, Hartley JW, Klinken SP, Ruscetti SK, Morse $\mathrm{HC}$, 3rd. v-cbl, an oncogene from a dual-recombinant murine retrovirus that induces early B-lineage lymphomas. Proc Natl Acad Sci USA 1989; 86:1168-1172.

4 Hamilton E, Miller KM, Helm KM, Langdon WY, Anderson SM. Suppression of apoptosis induced by growth factor withdrawal by an oncogenic form of c-Cbl. J Biol Chem 2001; 276:9028-9037.

5 Sinha S, Jancarik J, Roginskaya V, et al. Suppression of apoptosis and granulocyte colony-stimulating factor-induced differentiation by an oncogenic form of Cbl. Exp Hematol 2001; 29:746-755.

6 Widmann C, Gibson S, Johnson GL. Caspase-dependent cleavage of signaling proteins during apoptosis. A turn-off mechanism for anti-apoptotic signals. J Biol Chem 1998; 273:7141-7147.

7 Denis G, Mandard S, Humblet C, et al. Nuclear localization of a new c-cbl related protein, CARP 90, during in vivo thymic apoptosis in mice. Cell Death Differ 1999; 6:689-697.

8 Corsois L, Quatannens B, Dumont P, et al. Association of a new c-Cbl related protein with the very first stages of apoptosis induction. Cancer Detect Prev 2002; 26:93-104.

9 Thien CB, Langdon WY. Negative regulation of PTK signalling by Cbl proteins. Growth Factors 2005; 23:161-167. 
10 Hanlon A, Jang S, Salgame P. Cbl-b differentially regulates activation-induced apoptosis in $\mathrm{T}$ helper 1 and $\mathrm{T}$ helper 2 cells. Immunology 2005; 116:507-512.

11 Kaabeche K, Guenou H, Bouvard D, et al. Cbl-mediated ubiquitination of alpha5 integrin subunit mediates fibronectindependent osteoblast detachment and apoptosis induced by FGFR2 activation. $J$ Cell Sci 2005; 118:1223-1232.

12 El Chami N, Ikhlef F, Kaszas K, et al. Androgen-dependent apoptosis in male germ cells is regulated through the protooncoprotein Cbl. J Cell Biol 2005; 171:651-661.

13 Xia Z, Dickens M, Raingeaud J, Davis RJ, Greenberg ME. Opposing effects of ERK and JNK-p38 MAP kinases on apoptosis. Science 1995; 270:1326-1331.

14 Davis RJ. Signal transduction by the JNK group of MAP kinases. Cell 2000; 103:239-252.

15 Ham J, Eilers A, Whitfield J, Neame SJ, Shah B. c-Jun and the transcriptional control of neuronal apoptosis. Biochem Pharmacol 2000; 60:1015-1021.

$16 \mathrm{Xu}$ Z, Maroney AC, Dobrzanski P, Kukekov NV, Greene LA. The MLK family mediates c-Jun N-terminal kinase activation in neuronal apoptosis. Mol Cell Biol 2001; 21:4713-4724.

$17 \mathrm{Xu} \mathrm{Z,} \mathrm{Kukekov} \mathrm{NV,} \mathrm{Greene} \mathrm{LA.} \mathrm{POSH} \mathrm{acts} \mathrm{as} \mathrm{a} \mathrm{scaffold} \mathrm{for}$ a multiprotein complex that mediates JNK activation in apoptosis. EMBO J 2003; 22:252-261.

18 Kukekov NV, Xu Z, Greene LA. Direct interaction of the molecular scaffolds POSH and JIP is required for apoptotic activation of JNKs. J Biol Chem 2006; 281:15517-15524.

$19 \mathrm{Xu}$ Z, Kukekov NV, Greene LA. Regulation of apoptotic cJun N-terminal kinase signaling by a stabilization-based feedforward loop. Mol Cell Biol 2005; 25:9949-9959.

20 Mota M, Reeder M, Chernoff J, Bazenet CE. Evidence for a role of mixed lineage kinases in neuronal apoptosis. J Neurosci 2001; 21:4949-4957.

21 Maroney AC, Finn JP, Bozyczko-Coyne D et al. CEP-1347 (KT7515), an inhibitor of JNK activation, rescues sympathetic neurons and neuronally differentiated PC12 cells from death evoked by three distinct insults. J Neurochem 1999; 73:19011912.

22 Deckwerth TL, Johnson EM Jr. Temporal analysis of events associated with programmed cell death (apoptosis) of sympathetic neurons deprived of nerve growth factor. $J$ Cell Biol 1993; 123:1207-1222.

23 Zeng S, Xu Z, Lipkowitz S, Longley JB. Regulation of stem cell factor receptor signaling by $\mathrm{Cbl}$ family proteins $(\mathrm{Cbl}-\mathrm{b} /$ c-Cbl). Blood 2005; 105:226-232.

24 Galisteo ML, Dikic I, Batzer AG, Langdon WY, Schlessinger J. Tyrosine phosphorylation of the c-cbl proto-oncogene protein product and association with epidermal growth factor (EGF) receptor upon EGF stimulation. J Biol Chem 1995; 270:20242-20245.

25 Kassenbrock CK, Anderson SM. Regulation of ubiquitin protein ligase activity in c-Cbl by phosphorylation-induced conformational change and constitutive activation by tyrosine to glutamate point mutations. J Biol Chem 2004; 279:2801728027.

26 Ghahremani MH, Keramaris E, Shree T, et al. Interaction of the c-Jun/JNK pathway and cyclin-dependent kinases in death of embryonic cortical neurons evoked by DNA damage. J Biol Chem 2002; 277:35586-35596.
27 Tenneti L, D'Emilia DM, Troy CM, Lipton SA. Role of caspases in N-methyl-D-aspartate-induced apoptosis in cerebrocortical neurons. J Neurochem 1998; 71:946-959.

28 Wilhelm M, Xu Z, Kukekov NV, Gire S, Greene LA. Proapoptotic Nix activates the JNK pathway by interacting with POSH and mediates death in a Parkinson disease model. J Biol Chem 2007; 282:1288-1295.

29 Xu Z, Sproul A, Wang W, Kukekov N, Greene LA. Siah1 interacts with the scaffold protein POSH to promote JNK activation and apoptosis. J Biol Chem 2006; 281:303-312.

30 Diener K, Wang XS, Chen C, et al. Activation of the c-Jun Nterminal kinase pathway by a novel protein kinase related to human germinal center kinase. Proc Natl Acad Sci USA 1997; 94:9687-9692.

$31 \mathrm{Wu}$ S, Loke HN, Rehemtulla A. Ultraviolet radiation-induced apoptosis is mediated by Daxx. Neoplasia 2002; 4:486-492.

32 Levkowitz G, Waterman H, Ettenberg SA, et al. Ubiquitin ligase activity and tyrosine phosphorylation underlie suppression of growth factor signaling by c-Cbl/Sli-1. Mol Cell 1999; 4:1029-1040.

33 Ettenberg SA, Magnifico A, Cuello M, et al. Cbl-b-dependent coordinated degradation of the epidermal growth factor receptor signaling complex. J Biol Chem 2001; 276:27677-27684.

34 Arevalo JC, Waite J, Rajagopal R, et al. Cell survival through Trk neurotrophin receptors is differentially regulated by ubiquitination. Neuron 2006; 50:549-559.

35 Biswas SC, Shi Y, Sproul A, Greene LA. Pro-apoptotic Bim induction in response to nerve growth factor deprivation requires simultaneous activation of three different death signaling pathways. J Biol Chem 2007; 282:29368-29374.

36 Gallo KA, Johnson GL. Mixed-lineage kinase control of JNK and p38 MAPK pathways. Nat Rev Mol Cell Biol 2002; 3:663-672.

37 Bustelo XR, Crespo P, Lopez-Barahona M, Gutkind JS, Barbacid M. Cbl-b, a member of the Sli-1/c-Cbl protein family, inhibits Vav-mediated c-Jun N-terminal kinase activation. Oncogene 1997; 15:2511-2520.

38 Miura-Shimura Y, Duan L, Rao NL, et al. Cbl-mediated ubiquitinylation and negative regulation of Vav. J Biol Chem 2003; 278:38495-38504.

39 Leung IW, Lassam N. Dimerization via tandem leucine zippers is essential for the activation of the mitogen-activated protein kinase kinase kinase, MLK-3. J Biol Chem 1998; 273:32408- 32415.

40 Nihalani D, Merritt S, Holzman LB. Identification of structural and functional domains in mixed lineage kinase dual leucine zipper-bearing kinase required for complex formation and stress-activated protein kinase activation. $\mathrm{J}$ Biol Chem 2000; 275:7273-7279.

41 Vacratsis PO, Gallo KA. Zipper-mediated oligomerization of the mixed lineage kinase SPRK/MLK-3 is not required for its activation by the GTPase cdc 42 but is necessary for its activation of the JNK pathway. Monomeric SPRK L410P does not catalyze the activating phosphorylation of Thr258 of murine mitogen-activated protein kinase kinase 4. J Biol Chem 2000; 275:27893-27900.

(Supplementary information is linked to the online version of the paper on the Cell Research website.) 